\title{
CXBN: A Blueprint for an Improved Measurement of the Cosmological X-Ray Background
}

L. M. Simms, J. G. Jeringan, B. K. Malphrus, R. McNeil, K. Z. Brown, T. G. Rose, L. Lim, J. A. Kruth, E. T. Thomas, M. S. Combs, R. T. Kroll, B. J. Cahall, T. T. Turba, B. L. Molton, M. M. Powell, J. F. Fitzpatrick, D. C. Graves, S. Anderson, L. R. Cominsky, K. S. Prasad, J. P. Doty, M. Wampler-Doty, S. D. Gaalema, S. Sun

July 9,2012

SPIE Optics and Photonics San Diego, CA, United States August 12, 2012 through August 16, 2012 
This document was prepared as an account of work sponsored by an agency of the United States government. Neither the United States government nor Lawrence Livermore National Security, LLC, nor any of their employees makes any warranty, expressed or implied, or assumes any legal liability or responsibility for the accuracy, completeness, or usefulness of any information, apparatus, product, or process disclosed, or represents that its use would not infringe privately owned rights. Reference herein to any specific commercial product, process, or service by trade name, trademark, manufacturer, or otherwise does not necessarily constitute or imply its endorsement, recommendation, or favoring by the United States government or Lawrence Livermore National Security, LLC. The views and opinions of authors expressed herein do not necessarily state or reflect those of the United States government or Lawrence Livermore National Security, LLC, and shall not be used for advertising or product endorsement purposes. 


\title{
CXBN: A Blueprint for an Improved Measurement of the Cosmological X-Ray Background
}

\author{
Lance M. Simms ${ }^{a}$, J. Garrett Jernigan ${ }^{b}$, Benjamin K. Malphrus ${ }^{c}$, Roger McNeil ${ }^{c}$, Keven Z. \\ Brown $^{c}$, Tyler G. Rose ${ }^{c}$, Luke Lim ${ }^{c}$, Steve Anderson ${ }^{d}$, Jeffrey A. Kruth ${ }^{c}$, John P. Doty ${ }^{e}$, \\ Matthew Wampler-Doty ${ }^{e}$, Lynn R. Cominsky ${ }^{d}$, Kamal S. Prasad ${ }^{d}$, Eric T. Thomas ${ }^{c}$, Michael \\ S. Combs ${ }^{c}$, Robert T. Kroll ${ }^{c}$, Benjamin J. Cahall ${ }^{c}$, Tyler T. Turba ${ }^{c}$, Brandon L. Molton ${ }^{c}$, \\ Margaret M. Powell ${ }^{c}$, Jonathan F. Fitzpatrick ${ }^{c}$, Daniel C. Graves ${ }^{c}$, Stephen D. Gaalema ${ }^{f}$, \\ Shunming $\operatorname{Sun}^{f}$ \\ ${ }^{a}$ Lawrence Livermore National Laboratory, Livermore, CA, 94550 USA; \\ ${ }^{b}$ Space Sciences Laboratory, Berkeley, CA, 94720 USA; \\ ${ }^{c}$ Morehead State University: Space Science Center, Morehead, KY, 40351 USA; \\ ${ }^{d}$ Sonoma State University, Rohnert Park, CA 94928 USA; \\ eNoqsi Aerospace, Pine, CO 80470 USA; \\ fBlack Forrest Engineering, Colorado Springs, CO 80918 USA;
}

\begin{abstract}
A precise measurement of the Cosmic X-Ray Background (CXB) is crucial for constraining models of the evolution and composition of the universe. While many large, expensive satellites have measured the CXB as a secondary mission, there is still disagreement about normalization of its spectrum. The Cosmic X-Ray Background NanoSat (CXBN) is a small, low-cost satellite whose primary goal is to measure the CXB over its two-year lifetime. Benefiting from a low instrument-induced background due to its small mass and size, CXBN will use a novel, pixelated Cadmium Zinc Telluride (CZT) detector with energy resolution $<1 \mathrm{keV}$ over the range 1-60 keV to measure the CXBN with unprecedented accuracy. This paper describes CXBN and its science payload, including the GEANT4 model that has been used to predict overall performance and the backgrounds from secondary particles in Low Earth Orbit. It also addresses the strategy for scanning the sky and calibrating the data, and presents the expected results over the two-year mission lifetime.
\end{abstract}

Keywords: X-Ray Background, CZT Detectors, NanoSat, Hard X-Ray

\section{INTRODUCTION}

Measurements of the X-ray sky dating back to the 1960s using sounding rockets revealed a surprising cosmic background glow of X-ray emission. Subsequent measurements using a variety of platforms, including high altitude balloons and on-orbit X-ray astronomy telescopes revealed a variety of discrete cosmic sources and an unresolved, diffuse background radiation field. This Cosmic X-Ray Background (CXB), usually defined as the integrated emission in the soft and hard x-ray bands from about 2-100 keV, is an isotropic, extragalactic flux of $\mathrm{X}$-rays that bombard the upper atmosphere of our Earth.

Theorists believe they have largely solved the mystery of the CXB. It is generally agreed that the CXB arises from the radiation generated by point sources outside of our galaxy; in particular, emission from Active Galactice Nuclei (AGN). The AGN that produce the CXB are too distant to be detected in the lower end of the energy spectrum, i.e. by optical or infrared telecopes. While nearly $100 \%$ of the CXBN $<2 \mathrm{keV}$ is thought to be accounted for, the spectrum $>2 \mathrm{keV}$ is more elusive. It is believed that the primary contributor to the CXB in this energy range near is Compton Thick AGN (those AGN with a column density of $N_{H}<\sim 10^{24} \mathrm{~cm}^{-2}$ ). However, the number density of Compton Thick AGN at high redshift predicted by local field surveys is not enough to fully account for the intensity of the CXBN near its peak at $30 \mathrm{keV}$.

If one accepts the premise that the CXB is the signature of obscured AGN, then it follows that a precise measurement of the CXB spectrum in the $15-200 \mathrm{keV}$ energy range is crucial for understanding the nature and 
statistical properties of these objects. As Yoshihiro states, to constrain the average number density of Comptonthick AGNs in the universe, the absolute intensity of the CXB at $30 \mathrm{keV}$ is a critical parameter. ${ }^{1}$ Unfortunately, the measurements made to date disagree considerably.

A number of different missions have measured the CXBN. Some of the most notable are:

- The High Energy Astrophysics Observatory (HEAO) The A-2 Cosmic Ray Experiment and the A-4 Hard X-Ray and Gamma Ray instrument measured the X-Ray background. HEA0 A-4 had an energy resolution of about $15 \mathrm{keV}$ at $60 \mathrm{keV}^{2}$.

- Integral The SPI telescope on board the INTEGRAL satellite had an energy resolution of $\sim 1.5$ to 2.2 $\mathrm{keV}$ for energies in the $50-100 \mathrm{keV}$ range. ${ }^{3}$

- Swift-BAT The Swift Burst Alert Telescope (BAT) has an energy range of 15-150 keV and an energy resolution of $\sim 7 \mathrm{keV}$ over that range. ${ }^{4}$

While each of these instruments contained state-of-the-art technology at the time they were launched, dramatic improvement in soft and hard x-ray detection has taken place in the years since. Furthermore, current technology allows for smaller readout circuitry and lower power consumption, which opens the possibility for a small and inexpensive mission devoted entirely to measuring the Cosmic X-Ray background. The Cosmic X-Ray Background NanoSat (CXBN) is one such mission. CXBN is a $2 \mathrm{U}$ CubeSat that will house a hybrid CMOS pixelated Mercury Cadmium Telluride detector and measure the spectrum with an energy resolution of $1 \mathrm{keV}$ FWHM all the way up to $60 \mathrm{keV}$.

There are a number of reasons why a small satellite is an ideal platform for making a measurement of the CXBN spectrum. For one, the CXB is bright. There are approximately 4 photons $\mathrm{cm}^{-2} \mathrm{~s}^{-1} \mathrm{sr}^{-1}$ in the band from 10-100 keV, so a large collecting area is not required to obtain high signal-to-noise. Second, because the CXBN is isotropic and time-invariant, a satellite need not have precise attitude control or high time resolution or data rate to the ground. And lastly, the small amount of material in a nano-sat reduces contamination from charged particles or compton-scattered gamma-rays (particle rejection is not difficult with a finely pixelated detector, but radioistopes created within spacecraft materials are still a problem as their emission mimics radiation from the CXB).

The purpose of this paper is to describe the CXBN mission in fine detail. In the first section, a broad overview of the satellite and its anticipated timeline will be presented. A thorough description of the science payload and the CZT detector, including the instrumental response predicted by GEANT4 ${ }^{5}$ modeling will then follow. The last two sections will provide an overview of the observing and calibration strategy along with the expected results.

\section{OVERVIEW OF CUBESAT}

The CXBN, shown in Figure 1, is a $2 \mathrm{U}$ cube-satellite, or CubeSat. Each unit, or "U", is a $10 \times 10 \times 10 \mathrm{~cm}$ cube, making the entire satellite $20 \times 10 \times 10 \mathrm{~cm}$ in volume. Its upper half (furthest from the solar panels) consists largely of the scientific payload, which will be discussed in the following section. Its lower half is devoted to powering the main bus, communications, and the Attitude Determination and Control System (ADCS).

\subsection{Attitude Control}

As mentioned previously, studying the CXB does not require precise attitude determination or control, and there is no need for fast slew rates. This allows CXBN to use magnetorquers for both slewing and attitude stabilization. With a predicted orbit of $400 \mathrm{~km}$ perigee and $700 \mathrm{~km}$ apogee, the minimum and maximum magnetic fields that the satellite will encounter are roughly $2.284 \times 10^{-5}$ and $2.601 \times 10^{-5}$ Telsa, respectively. Three mutually perpendicular torquing coils, each having $\sim 230$ turns and a maximum current of $100 \mathrm{~mA}$ is enough to generate a magnetic moment of $0.166 \mathrm{~A} \cdot \mathrm{m}^{2}$, and a resultant torque of $\sim 4 \times 10^{-6} \mathrm{~N} \cdot \mathrm{m}$ about each axis. With these properties, Spacecraft dynamic simulations show that CXBN will be able to detumble in about 3000 seconds. 


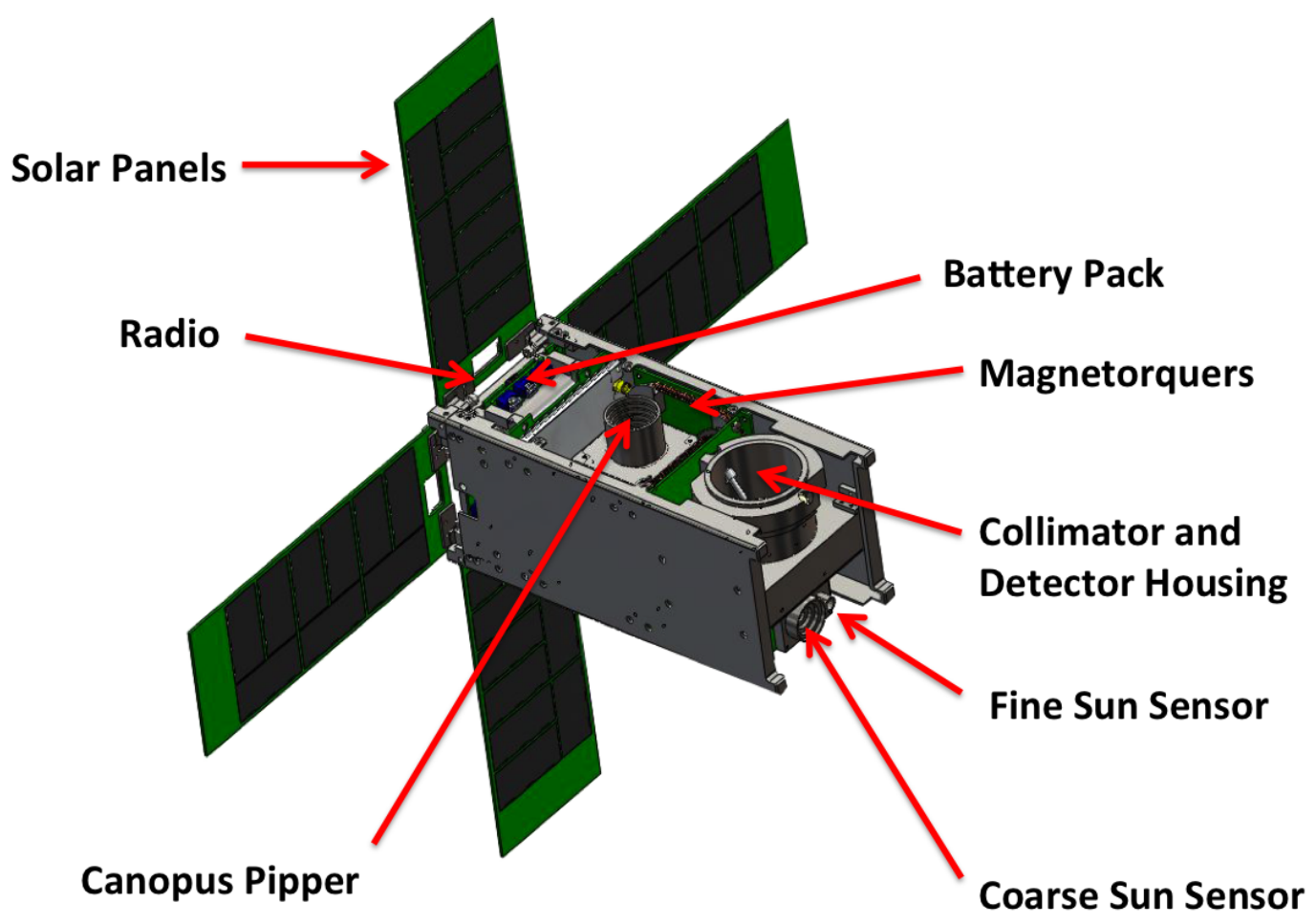

Figure 1. A detailed drawing of the CXBN Satellite. Each component is lableled.

\subsection{Attitude Determination and Pointing Strategy}

The CXBN has a number of attitude determination subsystems: MEMS gyros, magnetometers, a canopus pipper and a dual sun sensor package consisting of a medium and fine sun sensor. All of these components will be used in the the basic pointing and scanning strategy, which will be for the longer $z$ axis of the satellite to point at the sun, with the dual sun sensor system assuring that this axis remains fixed. The satellite will then spin about this axis at a rate of $\sim 1 / 6 \mathrm{~Hz}$. Since the payload collimator is aligned with the $x$ axis, the detector will sweep out a circle on the sky. Further details about data acquisition/storage and sky coverage will be discussed in Section 4 .

\subsection{Communication}

Two different radios are present on CXBN. One is a standard UHF link that will transmit at $437.525 \mathrm{MHz}$ and the other is an S-band radio capable of operating over the $2400-2410 \mathrm{MHz}$ range. The primary means of communication will be with a 21 meter Space Tracking Antenna at Morehead State University. With approximately four good passes per day (or roughly 2700 seconds of contact), a $\sim 27 \mathrm{Mb}$ /day data downlink is possible with the UHF system and $\sim 320 \mathrm{Mb}$ with S-Band. Both will be more than sufficient to transmit all of the hit information to the ground.

\section{SCIENCE PAYLOAD}

At its most basic level, the science payload of CXBN is an extremely simple system. Shown in Figure 2 (a GEANT4 model), it consists of a detector enclosure and collimator that shield and set the field of view for a pixelated CZT detector. There are no focusing or masking optics present, and no active shielding is used. Despite its simplicity, as will be shown in the following subsections, GEANT4 simulations show that this system is capable of measuring the CXB with minimal contamination from other background events. 


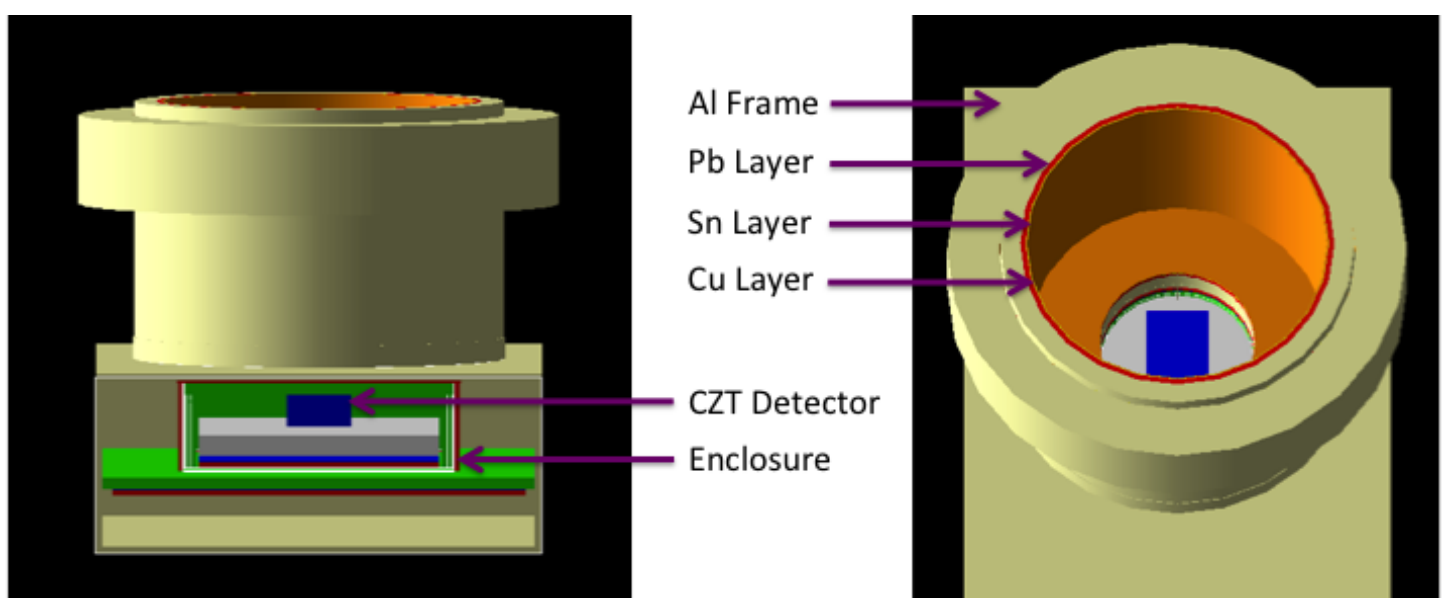

Figure 2. (Left) A side view of the CXBN science payload. The upper half contains the collimating cylinder, which has a graded-Z shield at its interior, and the lower half is where the detector and its surrounding enclosure, also made of the graded-z materials, are located. (Right) A top-down view of the payload highlighting the layers of the shield: $\mathrm{Pb}$ (red), Sn allow (yellow), and $\mathrm{Cu}$ (orange) from outside to in.

\subsection{Graded Z Shield for Collimator and Detector Enclosure}

As previously mentioned, CXBN benefits from having a low mass and small amount of material when it comes to radioisotopes and secondary particle production. However, shielding the detector is still very important. The purpose of the collimator and detector enclosure is to limit the detector Field of View (FOV) to a particular solid angle on the sky by blocking all particles coming from outside of that solid angle. But due to limitations on the mass of the satellite, the materials used for these structures cannot be arbitrarily thick. A large amount of effort was thus placed on determining the optimum materials and thicknesses for shielding, as well as the size of the collimator opening angle. Each will be discussed in turn.

\subsubsection{Collimator Field of View}

The collimator FOV is an extremely important parameter for the CXBN mission. It is set to balance Signalto-Noise-Ratio (SNR) from the CXB and contamination from bright X-ray sources. If it is too small, the signal from the CXB will too small. If it is too large, there will be too much contamination from bright X-ray point sources on the sky.

To optimize the FOV, simulated scans of the sky were performed using the HEAO A4 Source Catalog ${ }^{6}$ over a range of collimator "opening angles". * At each opening angle, the following quantities were calculated:

D - The number of counts/sec from the CXB in the energy range 10-100 keV. This is determined by multiplying the solid angle (in sr) by 5.25 counts $/ \mathrm{cm}^{2} / \mathrm{sr} / \mathrm{s}$.

$\mathbf{P}$ - The number of counts/sec from the particle background in Low Earth Orbit that are not rejected by the detector (more of this in the Section 5).

$\mathbf{S}$ - The number of counts/sec from bright x-Ray sources in the FOV, as determined by the HEAO A4 catalog.

\footnotetext{
* "Opening angle" is put in quotes because it is not an actual angle in the shape of the collimating cylinder; it is determined by the ratio of the cylinder height to its width. The angle goes as $\tan (H / d)$, where $H$ is the distance from the bottom of the sensitive layer of the detector to the top of the cylinder and $d$ is the maximum distance from an edge of the detector on the bottom surface to the cylinder wall on the opposite side.
} 


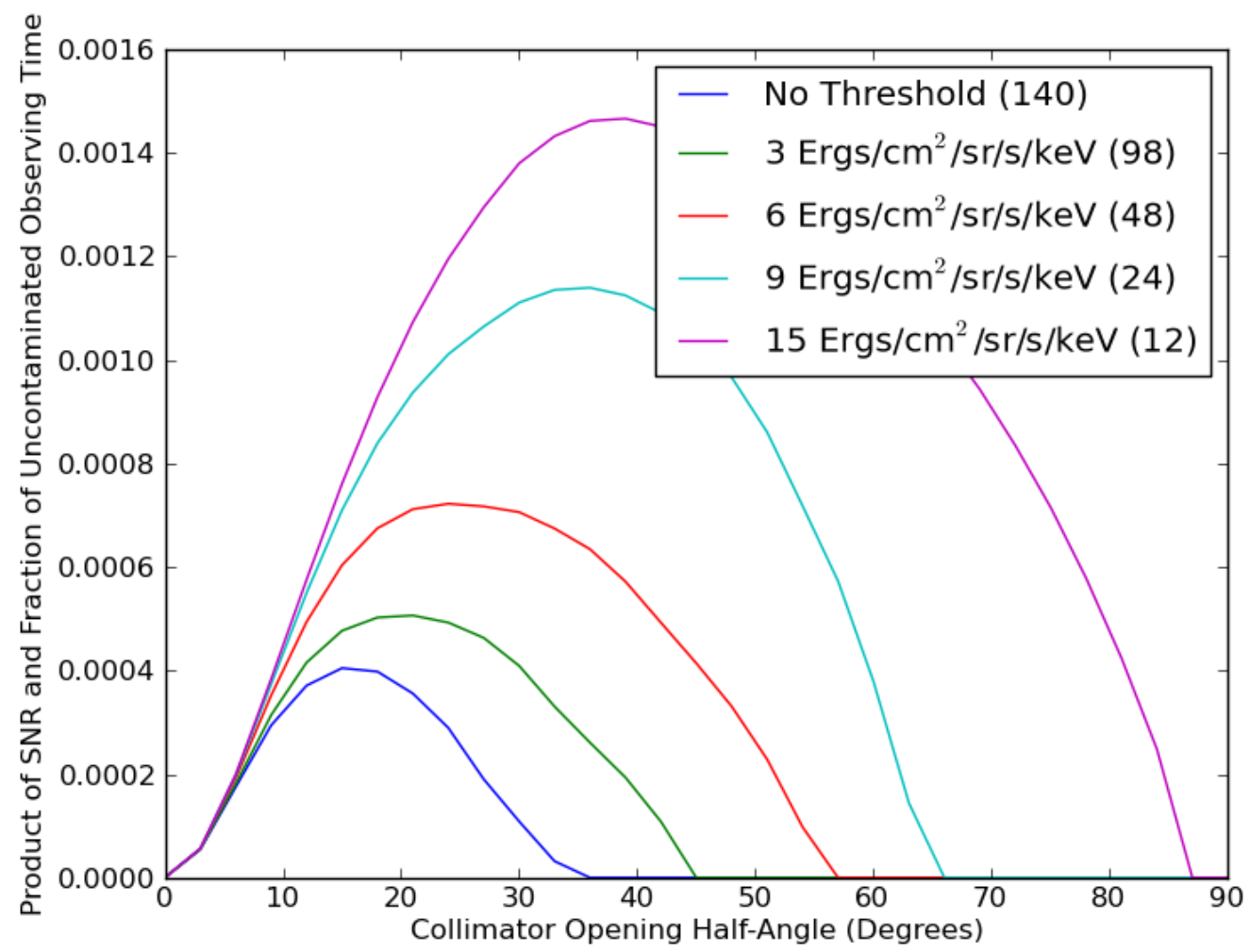

Figure 3. Curves showing the relative usefulness of various FOVs for the CXBN collimator. The y-axis is a product of the average SNR obtained while scanning the sky and the fraction of pointings that were contaminated by a source with energy greater than $E_{\text {upper }}$. Each color corresponds to a different $E_{\text {upper. }}$. The total number of sources on the sky with $E>E_{\text {upper }}$ is listed in parentheses in the legend.

and from this, the SNR was calculated as

$$
S N R=\frac{D}{\sqrt{D}+P+S}
$$

at each pointing. The average of the SNR at that particular opening angle was then determined, and along with this, the total fraction of pointings that were not contaminated by sources below an energy threshold $E_{\text {upper }}$. The product of these two numbers serve as a proxy for the usefulness of the opening angle.

Curves for a range of $E_{\text {upper }}$ are shown in Figure 3. Using a non-zero value of $E_{\text {upper }}$ allows for a higher SNR over a given period of time, but given the two-year lifetime of the mission, ample SNR can be achieved without allowing contamination from sources above $E_{\text {upper }}$. Thus, the angle of $\sim 18$ degrees was adopted for the CXBN FOV.

\subsubsection{Graded-Z Shielding}

To make the collimator and detector enclosure effective, the shield that shrouds them must block as many of the high-energy particles and photons in Low Earth Orbit as possible (outside of the FOV). Although lead is the most frequently used material for this purpose in a range of applications, making the shield entirely of it is not the most efficient method for stopping ionizing radiation. It turns out that a multi-layered shield of made of materials with successively lower atomic number $(\mathrm{Z})$ is more effective. The reason for this is that incident $\mathrm{X}$-rays and gamma-rays have a high probability of being absorbed in the lead and producing K-shell fluorescence 
Figure 4. A prototype for the Cadmium Zinc Telluride Detector used in CXBN. The ROIC that is bonded to the sensitive CZT layer has $600 \mathrm{um} \times 600$ um pixels.

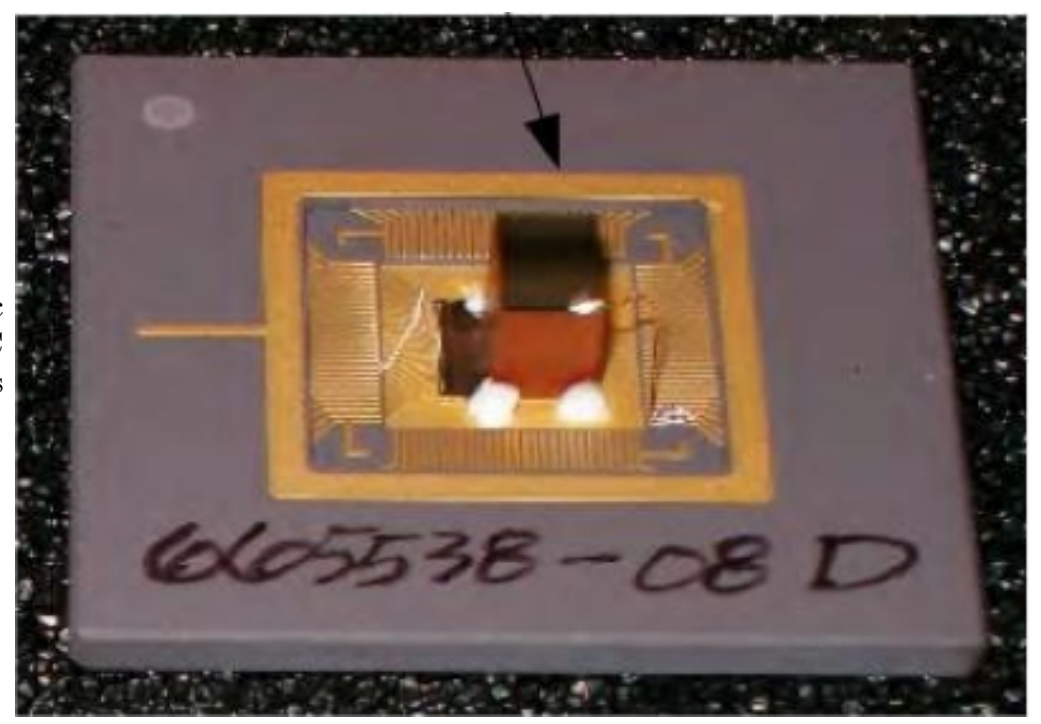

photons. Placing materials with lower $\mathrm{Z}$ after the lead layer allows these lower energy fluorescence photons to be further absorbed and degraded in energy before reaching the detector.

The materials chosen for the graded-Z shield of CXBN were based largely on the shielding used for the Burst Alert Telescope on the Swift satellite. ${ }^{7}$ However, Tantalum was not available, so the materials chosen were lead, tin, and copper, as shown in Figure 2. Using a mass allocation of about 200 grams, the shield geometry was created in GEANT4 such that the layer thicknesses could be altered easily between simulation runs. After sweeping through the 3-parameter space of thicknesses, it was determined that the optimal configuration was $0.95 \mathrm{~mm}$ of $\mathrm{Pb}, 0.19 \mathrm{~mm}$ of $\mathrm{Sn}$, and $0.06 \mathrm{~mm}$ of $\mathrm{Cu}$.

The shield significantly cuts down on the number of downshifted or "punch-through" $\mathrm{x}$-Rays that are registered in the detector. And for protons, electrons, He nuclei, etc., the spatial morphology of the charge distribution in the detector will be used to reject a large fraction that make it through the shield. In Section 5, the expected contamination levels from the backgrounds in Low Earth Orbit through the shield will be discussed.

\subsection{Cadmium Zinc Telluride (CZT) Detector}

Developed at Black Forrest Engineering, the CZT detector used in CXBN represents a large step forward in low-power detectors for space-based applications and is intended to fulfill future NASA missions in X-ray and Gamma-ray astronomy. It is comprised of an ultra-low power Read-Out Integrated Circuit (ROIC) that can be bump bonded to a sensitive Silicon sense layer for detection in the $2-30 \mathrm{keV}$ range or CZT for sensitivity over $5-600 \mathrm{keV}$. The pixels are $600 \mathrm{um} \times 600 \mathrm{um}$ and the format is $16 \mathrm{x} 32$, yielding a total sensitive area of $0.18 \mathrm{~mm}^{2}$. For CXBN, the thickness of the sensitive CZT layer is $1 \mathrm{~mm}$.

The ROIC is based on several key design concepts that represent an advance for X-ray detection over the use of active pixel sensors developed for longer wavelengths. Some of these concepts include:

(1) Smart pixels that autonomously detect X-rays and store the energy measurements in an analog sample and hold for subsequent readout.

(2) The front end of the pixel design is AC coupled with a threshold settable discriminator circuit. Each pixel only sees X-ray hits and only integrates dark current over a few microsecond window of time. Each pixel operates autonomously without the need for a global synchronous clock.

(3) Power is low because only hits above threshold are measured with an ADC (no zeros are readout). 


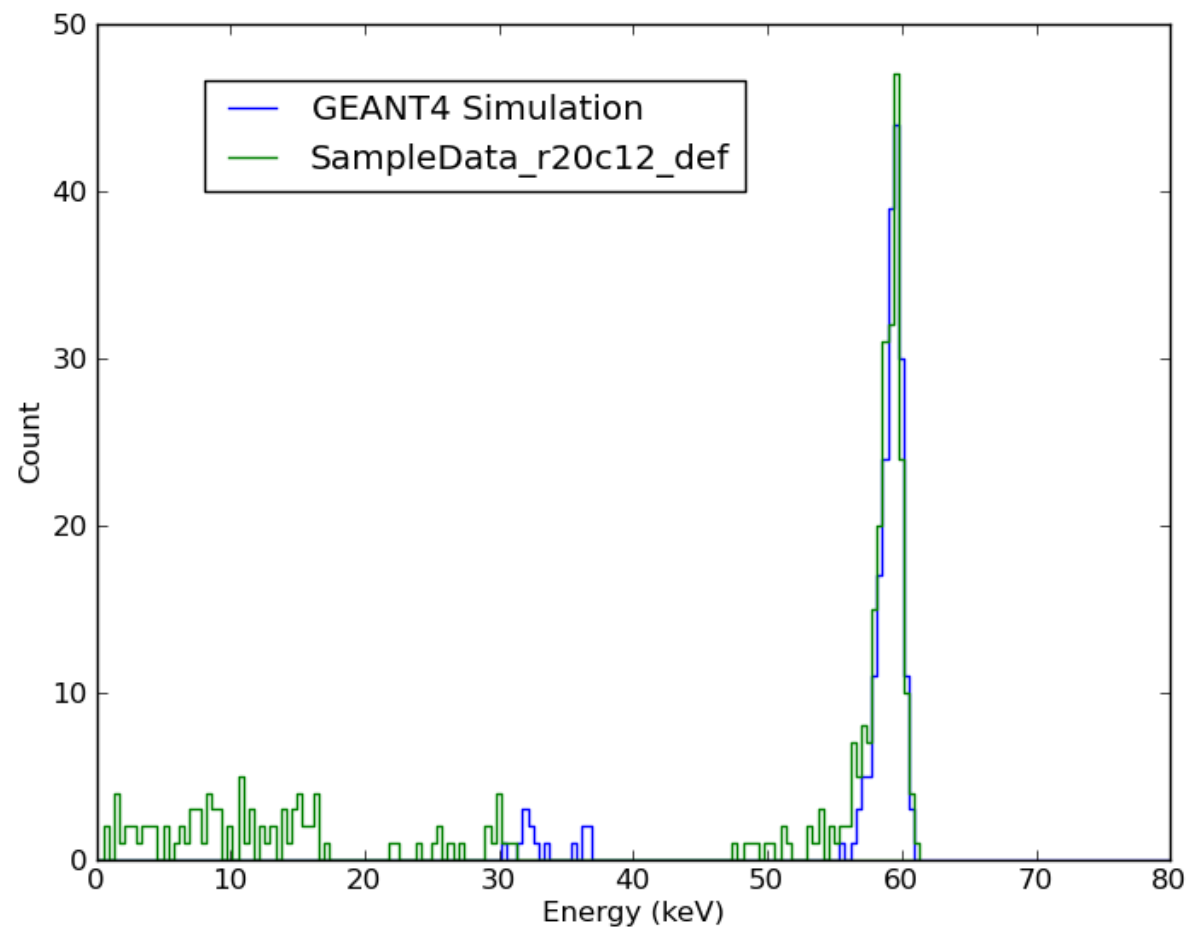

Figure 5. The spectrum obtained from an Am-241 source. The blue line shows the results from GEANT4 simulation and the green line shows real data obtained with the Blackforest $5 \mathrm{~mm}$ CZT device. Note that the escape peaks are not seen in the data because the discriminator threshold was not set high enough.

(4) If a pixel is hit then the neighbors of that pixel $(3 \times 3)$ also sample and store the charge even if below threshold.

(5) Time tagging to 1 microsecond assuming a low hit rate $(<10$ hits/s/cm2)

As one can see from these points, the array is very different from the typical hybrid CMOS detectors that are read out in a progressive scan fashion. Due to the fact that the detector dark current is negligible and the read noise is low, the array is able to achieve exquisite performance.

Additionally, GEANT4 simulations of the detector have shown that charge transport through the CZT layer of the Blackforest device is not hindered by crystal impurities or bulk defects, as is the case with most CZT detectors. The simulations used to model the CXBN detector incorporate GEANT4 to model the energy deposition of gamma and X-rays in the detector. Additional code then models the diffusion of charge to the surface along with the effect of read and fano-noise on the measurement obtained in each pixel. Results of a simulation where the detector was exposed to an Amerecium 241 source are shown in Figure 5. As one can see, with a read noise of 110 electrons, the energy resolution at $60 \mathrm{keV}$ matches up very well between the simulation and the data. The tail at the lower end of the peak is predicted by the diffusion of charge between pixels; it is not charge loss in the crystal. And even with the diffusion, the energy resolution is $\sim 1 \mathrm{keV}$ at this energy.

\section{METHODS FOR OBTAINING AND CALIBRATING DATA}

\subsection{Sky Scanning Strategy}

With the sun sensors pointing at the sun and the spacecraft spinning about the z-axis at a $\sim 1 / 6 \mathrm{~Hz}$ rate, the CZT detector will sweep out a great circle on the sky. Since the orbital, $T_{\text {orbit }} \sim 90$ minutes, is much greater 


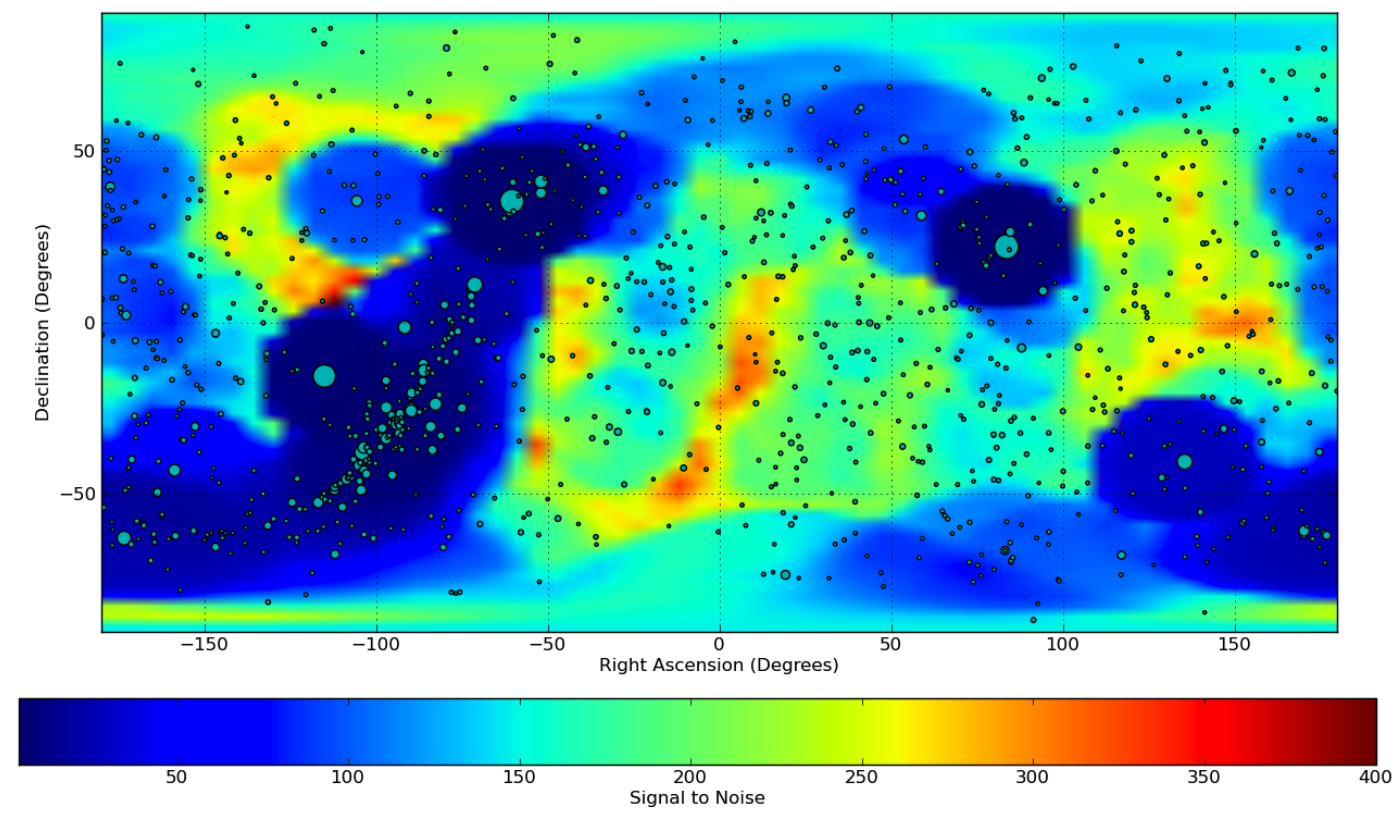

Figure 6. An all sky map showing the signal to noise that will be achieved over the course of one year. The cyan circles are X-ray sources catalogued by the BAT instrument aboard the SWIFT telescope. ${ }^{8}$ Their size is proportional to their flux.

than the spacecraft period of spin, $T_{\text {spin }}$, the detector will see roughly only one half of this great circle for each half of the orbit due to earth occultation. Over a full orbit, each half of the circle will be covered. And as the Right Ascension and Declination of the sun change over the course of the year, the great circle will sweep out a sphere on the sky.

Not all parts of the sky are equally useful for measuring the CXB because of X-ray point sources. The Signalto-Noise Ratio (SNR) depends strongly on what sources are present within the 18 degree FOV of the detector. Figure 6 shows the SNR that will be achieved over the course of a year at each point on the sky. The SNR is calculated over the energy range 10-60 keV, and assumes that all X-ray sources detected by the SWIFT BAT telescope are noise sources. Noise from unrejected cosmic rays has also been incorporated into the calculation.

As one can see, there are certain areas of the sky that provide a much higher SNR than others. The ecliptic poles provide strong signal because of repeated visits and the Vernal Equinox $\left(\mathrm{RA}=0^{\circ}, \mathrm{DEC}=0^{\circ}\right)$ also does because a lack of bright sources. The poles will provide about 1.5 million seconds of data while the area along the ecliptic will yield about 300,000 seconds in the first year alone.

\subsection{Detector Calibration}

To determine the normalization of the CXB spectrum and obtain the proper conversion gain of the detector, onorbit calibration is essential. The CXBN will have two separate Americium-241 sources on board to accomplish these two tasks.

The first calibration source will be used for gain calibartion. It is a 1 microCurie pellet mounted directly above the corner of the detector at a height of $2 \mathrm{~cm}$. It is housed in a cylindrical lead tube of height $1 \mathrm{~cm}$ and diameter $0.1 \mathrm{~cm}$, providing a collimated beam of X-rays. With this geometry, there are approximately 8 hits/second on the corner pixels of the CZT detector from the $59.5 \mathrm{keV}$ line and 9 hits/second from the $13.9 \mathrm{keV}$ line. These two lines will provide gain calibration over the primary range of interest: $10-60 \mathrm{keV}$.

The other source will also be a 1 microCurie Am-241 pellet. This one will be used to monitor the quantum efficiency of the detector on orbit. It will be mounted in a slightly shorter cylinder opposite to the gain source 
such that it illuminates the entire detector with X-rays. On the ground, an identical Am-241 pellet and CZT detector setup reference setup will serve to measure any deterioration in the sensitivity over time.

\section{SEPARATING SIGNAL FROM NOISE}

The data that is sent to the ground will consist of a series of counts for $3 \times 3$ pixel regions, e.g. nine numbers for each energy deposition that exceeds the discriminator threshold. Of course, this data will be contain many contaminants: X-ray point sources, Helium nuclei, electrons, positrons, etc. With the precise time-tagging, many of these contaminants will be vetoed due to their extended morphology (electrons and protons tend to leave extended tracks unless they are normally incident on the detector). However, some of them will be completely indiscriminable from X-ray hits.

Background particles in CXBN in the energy range 10-100 keV will result from the interaction of primary cosmic ray particles (electrons, positrons, protons and helium nuclei) in the materials of the CXBN instrument producing secondary particles that are detected in the CZT array. By their large energy deposits in the CZT, oninteractive primary cosmic rays are easily recognized and excluded. However, the secondary particles produced in the detector materials are often in the energy range of interest for the cosmic diffuse x-rays and must be subtracted from the cosmic $\mathrm{x}$-ray signal rate.

These backgrounds in CXBN were simulated using GEANT4. The materials of the collimator, shielding, and other structures near the CZT array were defined. Cosmic ray protons, electrons, positrons, and helium nuclei were generated with isotropic distribution about the CXBN detector. A total of 500 million positrons and electrons with energies ranging from $10 \mathrm{MeV}$ to $2 \mathrm{GeV}$ were generated, as were 1.1 billion protons with energies from $70 \mathrm{MeV}$ to $20 \mathrm{GeV}$, and 1 billion helium nuclei from $50 \mathrm{MeV}$ to $1 \mathrm{GeV}$, and protons with energies ranging from $70 \mathrm{keV}$ to $20 \mathrm{GeV}$. Energy deposited by secondary particles were recorded in the sensitive CZT region.

The rate of cosmic ray primaries was estimated using measurements in low earth orbit. Rates for electrons and positrons, ${ }^{9}$ protons, ${ }^{10}$ and helium nuclei ${ }^{11}$ as a function of geomagnetic latitude were combined with the projected orbits of CXBN to produce the absolute flux of cosmic ray primaries for CXBN when it is not in the region of extremely high particle flux. This rate was then combined with the probability that the primary cosmic ray particle produces a secondary particle in the energy range 10-100 keV to obtain a prediction for the absolute rate. This can be compared with the rate of signal x-rays that have been generated with an isotropic distribution and simulated by the same GEANT4 detector software and combined with a measured rate from Moretti et al. ${ }^{12}$ to produce a prediction of the absolute rate of cosmic diffuse background x-rays in CXBN.

Figure 7 shows the expected number secondary particles per keV energy bin from primary electrons and positrons, protons and helium particles as a function of energy deposited in the CZT, in 106 seconds of CXBN operations. Also shown is the expected number of cosmic ray x-ray per keV energy bin. The overall expected rate of particles depositing less than $100 \mathrm{keV}$ in the CZT is $2.46 \mathrm{~Hz}$, with the fraction coming from secondary particles from cosmic ray electrons, positrons, protons and helium nuclei ranging from $0.5 \%$ at $10 \mathrm{keV}$ to $15 \%$ at $100 \mathrm{keV}$. In the range $30-50 \mathrm{keV}$ deposited energy the expected background fraction is $2-4 \%$. The features seen at $25,28,73,75,85$, and $87 \mathrm{keV}$ correspond to $\mathrm{K}_{\alpha 1} 1, \mathrm{~K}_{\alpha 2}$, and $\mathrm{K}_{\beta 1} \mathrm{x}$-ray fluorescence transitions in $\mathrm{Pb}$ and $\mathrm{Sn}$ of the graded shielding in the structures surrounding the CZT. These lines may serve as secondary calibration sources for the experiment.

Further analysis of the x-ray rate in Figure 7 has determined that in the region $30-50 \mathrm{keV}, 85-89 \%$ of the $\mathrm{x}$-rays in the CZT are from non-interacting x-rays traveling within the angular subtended by the collimator into the CZT, 5-7\% are from x-rays that penetrate the shielding materials to deposit their energy in the CZT, and $5-8 \%$ are the result of secondary x-rays produced by primary x-rays interacting in the shielding materials.

\section{CONCLUSION}

The Cosmic X-Ray Background Nanosat is an extremely low-cost mission that will deliver big science. Armed with a novel Cadmium Zinc Telluride detector, it will scan the sky and measure the Cosmic X-Ray Background with excellent signal-to-noise. GEANT4 simulations have been performed to predict performance of the detector in Low Earth Orbit. The simulations show that with the low mass and volume of the satellite, and the small area 


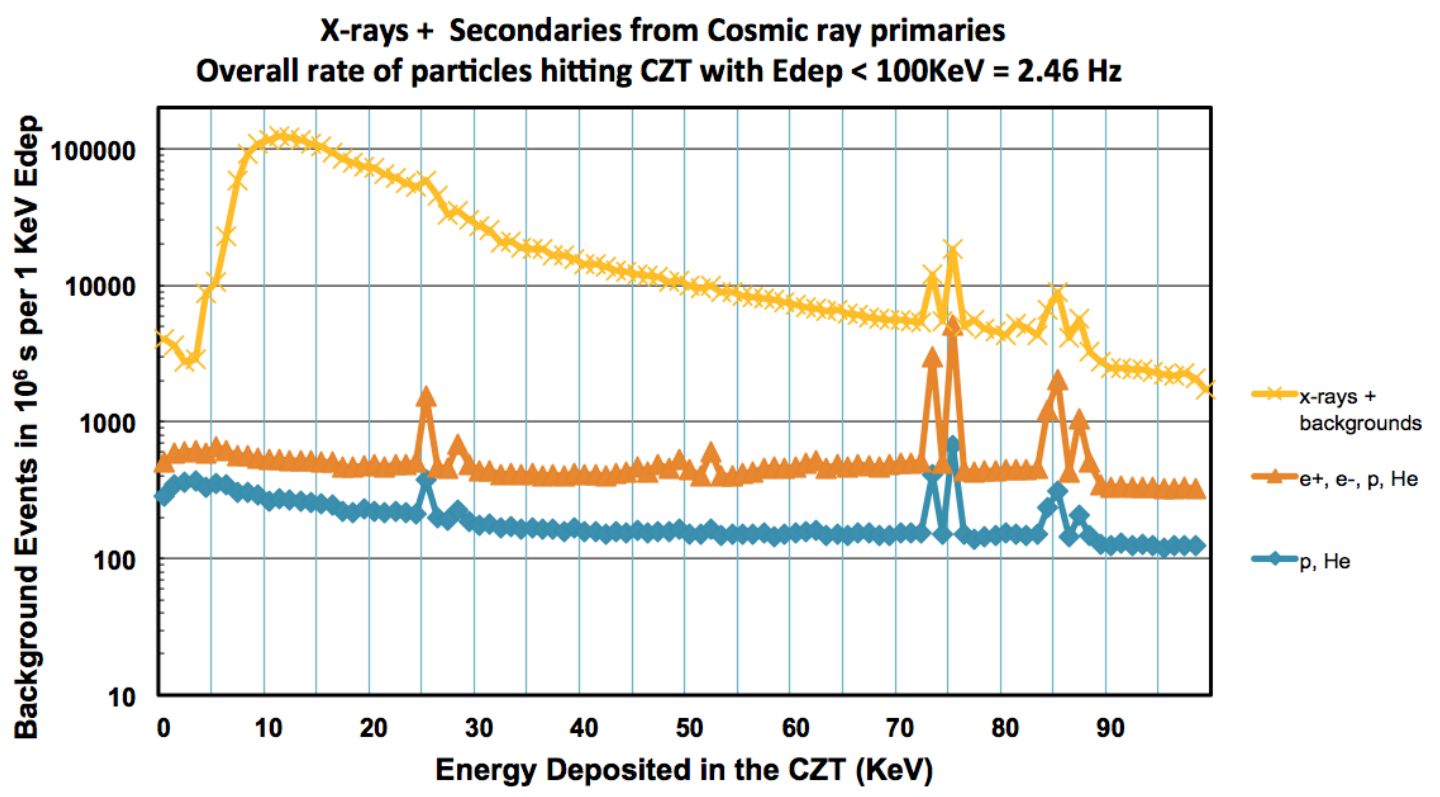

Figure 7. The plot shows a simulated energy spectrum obtained by CXBN. An isotropic flux of CXB X-rays were first allowed to impinge on the satellite; the signal measured by the CZT detector is shown in the yellow double arrows. The orange triangles represent the hits produced by electrons and positrons that could not be discriminated by morphology, and the blue diamonds represents the same for protons and Helium nuclei.

of the detector, excellent science results can still be achieved. Understanding the X-Ray Background is crucial to constraining cosmological models and understanding the universe around us. A successful launch of CXBN on August 2, 2012, will bring us one step closer to it.

\section{ACKNOWLEDGMENTS}

This work was performed under the auspices of the U.S. Department of Energy by Lawrence Livermore National Laboratory under Contract DE-AC52-07NA27344.

This document was prepared as an account of work sponsored by an agency of the United States government. Neither the United States government nor Lawrence Livermore National Security, LLC, nor any of their employees makes any warranty, expressed or implied, or assumes any legal liability or responsibility for the accuracy, completeness, or usefulness of any information, apparatus, product, or process disclosed, or represents that its use would not infringe privately owned rights. Reference herein to any specific commercial product, process, or service by trade name, trademark, manufacturer, or otherwise does not necessarily constitute or imply its endorsement, recommendation, or favoring by the United States government or Lawrence Livermore National Security, LLC. The views and opinions of authors expressed herein do not necessarily state or reflect those of the United States government or Lawrence Livermore National Security, LLC, and shall not be used for advertising or product endorsement purposes.

\section{REFERENCES}

[1] Ueda, Y., "Cosmic X-ray Background: An Old, yet New Story," Nature Physics 3, 450-451 (2007).

[2] Gruber, D. E., Matteson, J. L., Peterson, L. E., and Jung, G. V., "The Spectrum of Diffuse Cosmic Hard X-Rays Measured with HEAO 1," Apj 520, 124-129 (jul 1999).

[3] Churazov, E., Sunyaev, R., Revnivtsev, M., Sazonov, S., Molkov, S., Grebenev, S., Winkler, C., Parmar, A., Bazzano, A., Falanga, M., Gros, A., Lebrun, F., Natalucci, L., Ubertini, P., Roques, J.-P., Bouchet, L., Jourdain, E., Knödlseder, J., Diehl, R., Budtz-Jorgensen, C., Brandt, S., Lund, N., Westergaard, N. J., Neronov, A., Türler, M., Chernyakova, M., Walter, R., Produit, N., Mowlavi, N., Mas-Hesse, J. M., Domingo, A., 
Gehrels, N., Kuulkers, E., Kretschmar, P., and Schmidt, M., "INTEGRAL observations of the cosmic Xray background in the 5-100 keV range via occultation by the Earth," Astronomy and Astrophysics 467, 529-540 (May 2007).

[4] Barthelmy, S. D., Barbier, L. M., Cummings, J. R., Fenimore, E. E., Gehrels, N., Hullinger, D., Krimm, H. A., Markwardt, C. B., Palmer, D. M., Parsons, A., Sato, G., Suzuki, M., Takahashi, T., Tashiro, M., and Tueller, J., "The Burst Alert Telescope (BAT) on the SWIFT Midex Mission," Space Science Reviews 120, 143-164 (oct 2005).

[5] Agostinelli, S. et al., "GEANT4: A Simulation toolkit," Nucl.Instrum.Meth. A506, 250-303 (2003).

[6] Levine, A. M., Lang, F. L., Lewin, W. H. G., Primini, F. A., Dobson, C. A., Doty, J. P., Hoffman, J. A., Howe, S. K., Scheepmaker, A., Wheaton, W. A., Matteson, J. L., Baity, W. A., Gruber, D. E., Knight, F. K., Nolan, P. L., Pelling, R. M., Rothschild, R. E., and Peterson, L. E., "The HEAO 1 A-4 catalog of high-energy X-ray sources," Astrophysical Journal Supplement Series 54, 581-617 (apr 1984).

[7] Robinson, D. W., "Building a New Kind of Graded-Z Shield for Swift's Burst Alert Telescope," Proc. SPIE 4851 (2003).

[8] Baumgartner et al. Astrophysical Journal Supplement (2010).

[9] Alcaraz, J. et al., "Leptons in near earth orbit," Physics Letters B484, 10-22 (2000).

[10] Alcaraz et al., "Protons in near earth orbit," Physics Letters B 472, 215-226 (jan 2000).

[11] Alcaraz, J. et al., "Helium in near Earth orbit," Physics Letters B494, 193-202 (2000).

[12] Moretti, A., Pagani, C., Cusumano, G., Campana, S., Perri, M., et al., "A new measurement of the cosmic X-ray background," AIP Conferenece Proceedings 1126, 223-226 (2009). 